Short Note

\title{
Synthesis of 5-benzyl-2,6-dimethylpyridazin-3(2H)-one
}

\section{Nour-Eddine Benchat, Abderazak Benalla, Fouad El Kalai, Mohammed Ramdani and Said Daoui}

Laboratory of Chemistry applied and environment, University Mohamed I, Sciences Faculty, 60000 Oujda, Morocco

* Author to whom correspondence should be addressed; E-mai: n_benchat@yahoo.fr

Received: 11 September 2008 / Accepted : 5 November 2008 / Published: 23 November 2008

Keywords: Pyridazines, alkylation, microwave, anticancer activity.

Recentely, Rubat et al. [1] synthesized a series of products by alkylation of pyridazines, the authors showed that these products are good analgesics and have a low toxicity. In our ongoing reseach program, we have synthesized compound (II); it will be subjected to further pharmacological investigations, especially tests of its anticancer activity.<smiles>Cc1n[nH]c(=O)cc1Cc1ccccc1</smiles>

(I)

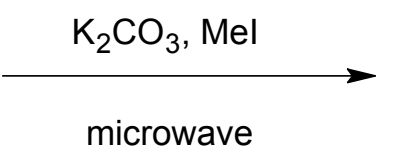

(II)<smiles>Cc1nn(C)c(=O)cc1Cc1ccccc1</smiles>

The product (II) was prepared from 5-benzyl-6-methylpyridazin-3(2H)-one (I) by solid-liquid PTC conditions without solvent [2]. To pyridazinone (I) (1.2 g, $5 \mathrm{mmol})$ were added potassium carbonate (0.692 g, $5 \mathrm{mmol})$, TBAB (0.3 g, $1 \mathrm{mmol})$ and methyl iodide $(0.73 \mathrm{~g}, 5 \mathrm{mmol})$. The mixture was placed in a pyrex tube which was then introduced into a Maxidigest MX 350 Prolabo microwave monomode reactor, fitted with a rotational system. At the end of the irradiation time $(10 \mathrm{~min}, 90 \mathrm{~W}$ irradiation power), the mixture was cooled to ambient temperature. The precipitate formed was filtered and washed with water, yield: $96 \%$ of (II). 
IR (KBr): $1663(\mathrm{CO}), 1591(\mathrm{C}=\mathrm{N}), 1430,1495(\mathrm{C}=\mathrm{C})$.

${ }^{1} \mathrm{H}$ NMR (300.14 MHz, $\left.\mathrm{CDCl}_{3}\right): \delta(\mathrm{ppm}): 2.20\left(\mathrm{~s}, 3 \mathrm{H}, \mathrm{CH}_{3}\right), 3.72\left(\mathrm{~s}, 3 \mathrm{H}, \mathrm{CH}_{3}\right), 3.81\left(\mathrm{~s}, 2 \mathrm{H}, \mathrm{CH}_{2}\right)$, $6.53(\mathrm{~s}, 1 \mathrm{H}, \mathrm{H}-4), 7.25$ (m, 5H, aromatic protons).

${ }^{13} \mathrm{C}$ NMR (75.48 MHz, $\left.\mathrm{CDCl}_{3}\right): \delta(\mathrm{ppm}) 19.12\left(\mathrm{CH}_{3}\right), 35.85\left(\mathrm{CH}_{2}\right), 39.67\left(\mathrm{NCH}_{3}\right), 127.66\left(\mathrm{CH}_{\text {aromatic }}\right)$, $127.87\left(\mathrm{CH}_{\text {aromatic }}\right), 129.32$ (2 $\left.\mathrm{CH}_{\text {aromatic }}\right), 129.51$ (2 $\left.\mathrm{CH}_{\text {aromatic }}\right), 135.66,145.25,146.52,160.63(\mathrm{C}=\mathrm{O})$.

Anal. Calcd for $\mathrm{C}_{13} \mathrm{H}_{14} \mathrm{~N}_{2} \mathrm{O}$ : $\% \mathrm{C}: 72.89 ; \% \mathrm{H}: 6.54 ; \%$ N: 13.08 . Found: $\% \mathrm{C}: 72.47 ; \% \mathrm{H}: 6.43 ; \% \mathrm{~N}$ : 12.72 .

\section{References}

1. Rubat, C.G.; Coudert, P.; Couquelet, J.; Bastide, P.; Bastide, J. Chem. Pharm. Bull. 1988, 36, 1558 .

2. De La Hoz, A.; Diaz-Ortiz, A.; Moreno, A. Chem. Soc. Rev. 2005, 34, 164.

(C) 2008 by the authors; licensee Molecular Diversity Preservation International, Basel, Switzerland. This article is an open-access article distributed under the terms and conditions of the Creative Commons Attribution license (http://creativecommons.org/licenses/by/3.0/). 